\title{
REFERENCES
}

1. van Rheenen PF, Van de Vijer E, Fidler V. Faecal calprotectin for screening of patients with suspected inflammatory bowel disease: diagnostic meta-analysis. BMJ 2010;341:c3369.

2. Whitehead SJ, Lee L, Fang Z, et al. Can faecal calprotectin reduce the demand for colonoscopy in patients with irritable bowel syndrome. Gut 2010;59(Suppl 1):A37.

\section{OC-098 FAECAL CALPROTECTIN (FC) IN SECONDARY CARE: UNNECESSARILY COMPLEX OR DEFINITELY HELPFUL?}

doi:10.1136/gut.2011.239301.98

M Srinivas, ${ }^{*}$ R Eyre, M Walsh, P Basumani, B Hoeroldt, P Willemse, K Bardhan Rotherham General Hospital, Rotherham, UK

Introduction Faecal calprotectin (FC) is a protein complex released from degraded neutrophils exuded through the gut wall into the lumen. Its levels are therefore likely to better reflect the presence and intensity of gut inflammation than our conventional inflammatory markers: $\mathrm{C}$ reactive protein (most commonly used by us), platelets, albumin, endoscopy, histopathology and imaging. FC values $(\mathrm{mg} / \mathrm{kg})$ suggest inflammatory status as <50:Nil; 50-100: possible; >100: likely; >1000: definite. The factors limiting its routine use include need for spot stool collection, which many patients dislike and the time involved for manual assay, hence higher cost. Is the 'help' in decision making worth the effort and cost?

Aim To test the utility of FC in the routine clinical setting of a gastroenterology unit in a UK DGH.

Methods Retrospective study of consecutive new and follow up patients who had FC (PhiCal ELISA test) done in our clinics between 10/2007 and 2/2009 ( $n=200)$. Patients were categorised into IBD-flare (relapse), IBD-active (persistent activity), IBD-remission and non-IBD (eg, IBS, abdominal pain, weight loss). The spot FC values are tabulated as median and range according to presence or absence of inflammation by conventional markers as above ( $\geq 1$ test done within 2 weeks in majority).

Results See table 1.

Conclusion FC levels $>500$ correlate well with flare and active IBD. Very low levels $(<7.8)$ reliably indicate lack of significant

Table 1 OC-098 FC in IBD and non-IBD patients

\begin{tabular}{|c|c|c|c|c|c|c|}
\hline \multirow[b]{2}{*}{ Condition } & \multicolumn{3}{|c|}{ Inflammation by conventional markers } & \multicolumn{3}{|c|}{ No inflammation by conventional markers } \\
\hline & $\mathbf{N}$ & Median FC (mg/kg) & FC range & $\mathbf{N}$ & Median FC (mg/kg) & FC range \\
\hline IBD-flare $(n=28)$ & 26 & 1117 & $56-4580$ & 2 & 777 & $97-1458$ \\
\hline IBD-remission ( $\mathrm{n}=38$ ) & 20 & 112 & $7.8-2500$ & 18 & 50 & $6-95$ \\
\hline Non-IBD symptomatic $(\mathrm{n}=98)$ & 25 & 79 & $7.8-2500$ & 73 & 36 & $4-1683$ \\
\hline
\end{tabular}

GI inflammation. Levels $>7.8$ in non-IBD patients (majority of new referrals) may indicate ongoing GI inflammation in a few and hence the need to investigate fully rather than assume functional GI disease as the FC levels are within the published "normal" range. Raised FC in IBD-remission may signify ongoing silent inflammation with potentially significant influence on long-term prognosis (an area to be explored).

Discussion This retrospective pilot study in our unit suggests that the effort and cost of FC is exceeded by the help it offers. It is of definite help when levels are very high or very low. We need to assess systematically the disease correlation with moderate FC elevation to develop local normal values.

\section{Competing interests None.}

